\title{
Use of cotton bud in Malaysia
}

\author{
Sakina Bt Ghauth*, Rajagopalan Raman and Chong Aun Wee \\ Department of Otorhinolaryngology, Universiti Malaya Medical Centre, Malaysia
}

\begin{abstract}
Objective: Cotton bud is a product that is commonly used in Malaysia. The aim of the study is to discover the prevalence of cotton bud usage in the ear by Malaysians. Methods: Samples were selected randomly to answer a standard questionnaire.

Results: Out of 300 subjects, cotton bud is used to clean ears in 195(65\%) subjects, using both curette and cotton buds in 65 (21.7\%) subjects, using curette $36(12 \%)$ subjects and only $4(1.3 \%)$ subjects consulted doctor to clean their ears.

Conclusion: Cotton bud is use widely for ear cleaning. Many subjects were not aware that the use of cotton bud in ear might lead to serious ear problems. Public and health provider need to be educated regarding this matter so that cotton bud related ear problem can be reduced.
\end{abstract}

\section{Introduction}

Historically, the cotton bud was invented in the 1920s by Leo Gerstenzang after he attached wads of cotton to toothpicks [1,2]. Nowadays, cotton bud is a product that is widely used, easily available at local stores and is a common item found in every household in Malaysia. It is used for multiple purposes such as medical purpose, cleaning, cosmetic application and arts. However, it is most commonly used in ear for cleaning, removing of ear wax, to relieve ear itchiness, aural toilet in discharging ears and sometimes as a habit [3].

Curette or ear pick is another instrument use for ear cleaning. It is a commonly used item and preferred for ear wax removal in East Asia, whose dry type of ear wax can be easily removed using the tool [4]. It can be used individually or by another person (Figure 1). The person having his/her ears cleaned often lies or bends down with his/her head in the lap of the person doing the cleaning. The cleaning of ears is often performed by a parent on a child or, among adults, by one's partner [5]. It may also be performed by professional (non-medical) ear cleaners on the streets of cities in countries such as China, Japan and Korea [5].

\section{Material and methods}

This was a prospective survey using a standard questionnaire (Figure 2). The samples were selected randomly from age 13 years old and above. The subjects were required to complete the questionnaire form, which consisted of two main parts. In the first part, the subjects were asked regarding their personal data i.e. age, sex and ear problem. The second part was related to their perception regarding ear wax and method used to clean the ear.

\section{Results}

From the study, 300 samples were collected. The age ranges from 13 till 59 years old with mean age of 23.77 years old. There are 37 male and 263 females.

Out of 300 subjects, 172 subjects (57.3\%) responded that ear wax was a dirty substance, $78(26 \%)$ subjects responded as a normal product and $50(16.7 \%)$ subjects were not sure.

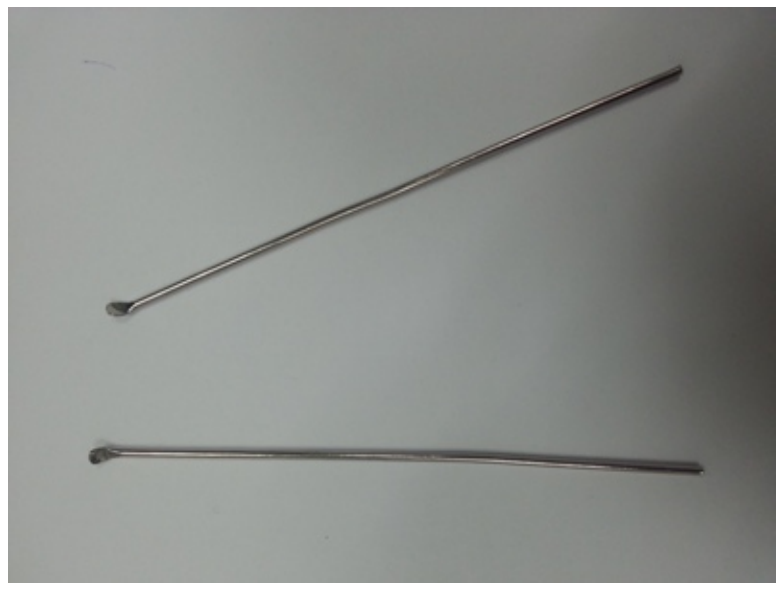

Figure 1. Curette

$204(68 \%)$ of the subjects felt that ear wax is a dirty substance and needs to be removed.

$40(13.3 \%)$ subjects felt that it is alright to have ear wax as long as it does not block the ear.

29(9.7\%) subjects felt that it is normal to have ear wax in ear.

$15(5 \%)$ subjects felt that it is alright to have ear wax and can be left alone as long as it serves its function.

12(4\%) subjects find it dirty but can be left alone.

${ }^{\star}$ Correspondence to: Sakina Bt Ghauth, Department of otorhinolaryngology University Malaya Medical Centre, Lembah Pantai, 59100 Kuala Lumpur, Malaysia, Tel: 0183130131, 0136136878, E-mail: sakina.ghauth@gmail.com

Key words: cotton bud, tympanic membrane, Malaysia

Received: August 13, 2018; Accepted: October 24, 2018; Published: October 27,2018 


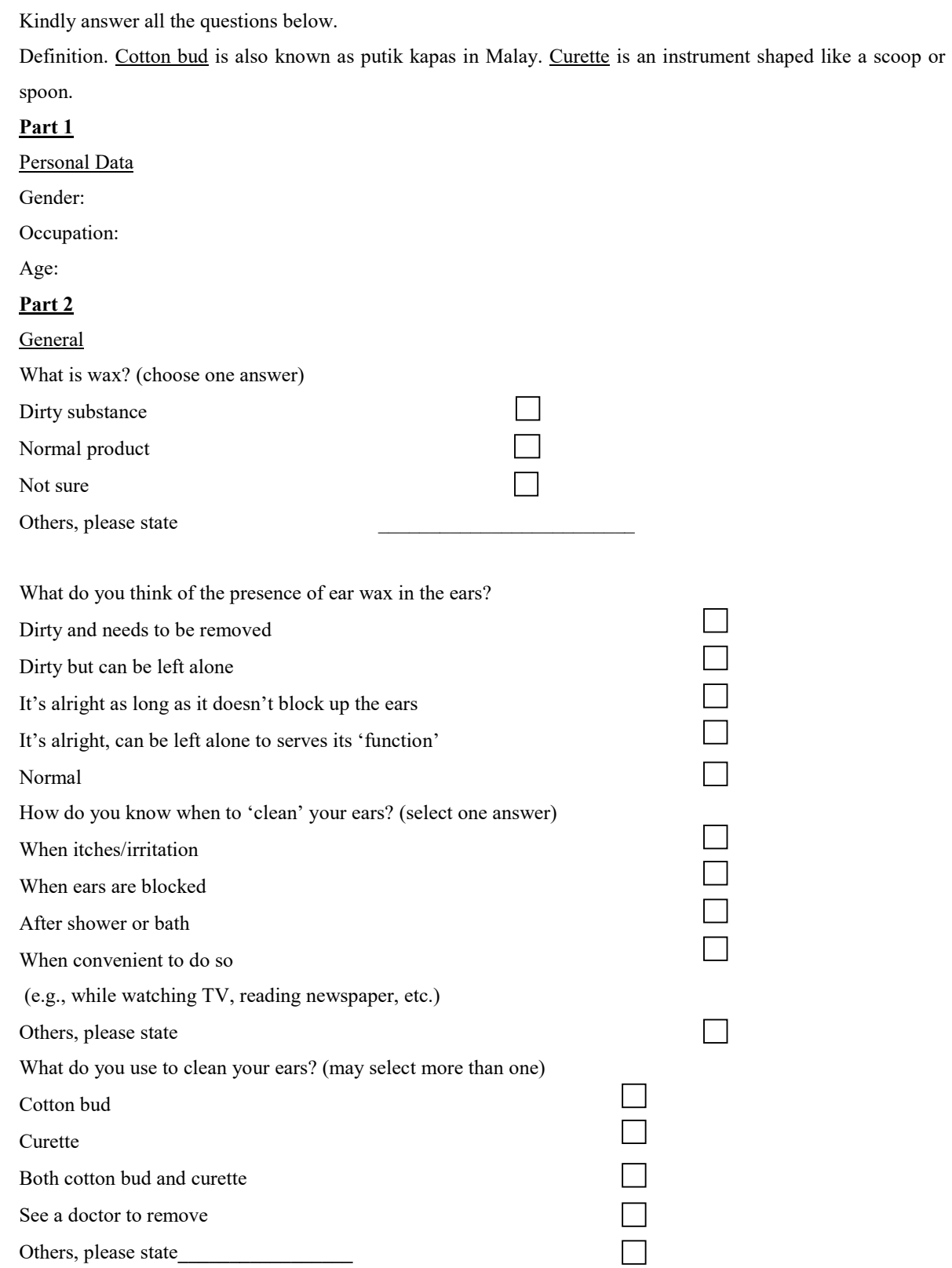

Figure 2. Questionnaire on usage of cotton bud in Malaysia

111(37\%) subjects cleaned their ears when there was irritation and itchiness.

108(36\%) subjects, cleaned their ears after a shower.

52(17.3\%) cleaned their ears when convenient (watching television or reading newspaper).

26(8.7\%) subjects cleaned their ears when there was ear blockage.

3 subjects did not state the condition where they needed to clean their ears.

Cotton buds is used to clean ears in 195(65\%) subjects.

Using both curette and cotton buds in 65 (21.7\%) subjects.

Using curette $36(12 \%)$ subjects.

Only $4(1.3 \%)$ subjects consulted doctor to clean their ears.

\section{Discussions}

Ear wax or cerumen is a mixture of ceruminous gland secretions, squames of epithelium, dust and other foreign debris [6]. It is expelled by epithelial migration from the tympanic membrane, aided by movements of the temporomandibular joint [7]. It protects the skin of the human ear canal, assists in cleaning and lubrication, and also provides some protection from infection, insects and water [8].

The use of cotton buds in the ears has widely been condemned worldwide by otolaryngologists [9]. Due to the frequent use of cotton bud in ear, ear injuries caused by cotton buds are commonly seen in ENT practice [9]. The first instances of medical concern over the use of cotton buds were in 1972 with reports of tympanic membrane perforation, otitis externa and cerumen impaction [10,11]. The most common accidental penetrating ear injury in children is cotton bud induced [12]. 
From this study, it is evident that there is public perception that ear wax is a dirty product and regular cleaning needs to be done. Especially in Malaysia, ear wax is called 'tahi telinga' in Malay language which is perceived as something dirty and need cleaning. This habit of cleaning the ear either with cotton bud or curette has been passed down generation to generation either by observation or introduced by their parents for the first time. Awareness of cotton bud/curettes related ear complications is an important public health issue. We recommend that cotton bud/curettes related complications and the notion of the self ear cleaning, needs to be raised not only to the public but also to medical professionals and manufacturers. As medical practitioners are the first line person for public to turn to when they have ear problems, the medical practitioners need to be updated and educated regarding this matter. They also need to participate in educating the public regarding self ear cleaning and its dangers. Safer method of aural toileting may also need promoting and if necessary, referrals to otorhinolaryngologist need to be done. Manufacturers of cotton bud also need to put labels in their packaging regarding warnings related to the danger of using cotton bud in the ear. On the other hand, little study has been done regarding the use of curette in the ear. Nevertheless, it can lead to similar complications in the ear as what the cotton bud does and likely more serious side effects. In conclusion, everyone has to play their role in minimizing the complication of cotton bud related ear problem by reducing or discontinuing the habit of using cotton bud, curette or another instrument in the ear.

\section{References}

1. Schueller R (1996) Cotton Swab.

2. Cotton Swab Q-tips History, Unilever Home and Personal Care 2007-2008.

3. Kumar S, Shamim A (2008) Use of cotton buds and its complications. J Surg Pak Int 13: $137-138$.

4. Overfield T (1985) Biologic variation in health and illness: race, age, and sex differences. Menlo Park, USA: Addison-Wesley Publishing.

5. Fumiko K, Laura. The Art of Ear Picking. SWINDLE Magazine.

6. Hanger HC, Mulley GP (1992) Cerumen: its fascination and clinical importance: a review. J R Soc Med 85: 346-349. [Crossref]

7. No authors listed (1972) Wax in the ear. Br Med J 4: 623-624. [Crossref]

8. Earwax at the American Hearing Research Foundation (2008), Chicago, USA.

9. Kravitz H, Neyhus AI, Dale DO, Laker HI, Gomberg RM, et al. (1974) The cottontipped swab: a major cause of ear injury and hearing loss. Clin Pediatr (Phila) 13: 965-970. [Crossref]

10. Robertson MS (1972) The misuse of cotton wool buds. N Z Med J 75: 37. [Crossref]

11. Barton RT (1972) Q-tip otalgia. JAMA 220: 1619. [Crossref]

12. Steele BD, Brennan PO (2002) A prospective survey of patients with presumed accidental ear injury presenting to a paediatric accident and emergency department Emerg Med J 19: 226-228. [Crossref]

Copyright: (C2018 Ghauth SB. This is an open-access article distributed under the terms of the Creative Commons Attribution License, which permits unrestricted use, distribution, and reproduction in any medium, provided the original author and source are credited. 